\title{
Article
}

\section{Dispersion and Re-aggregation of Particles in a Suspension Flowing in an Abrupt Contraction Channel}

\author{
Koji MASUdA ${ }^{*, \dagger}$, Katsuhito ARAKI ${ }^{*}$, Ruri HIDEMA ${ }^{* *}$, \\ Hiroshi SuZUKI $^{* * *}$, and Yoshiyuki KOMODA ${ }^{* * *}$ \\ "Department of Applied Chemistry, Kobe City College of Technology \\ 8-3, Gakuenhigashi, Nishi-ku, Kobe-shi, Hyogo 651-2194, Japan \\ ${ }^{* *}$ Organization of Advanced Science and Technology, Kobe University \\ 1-1, Rokkodai-cho, Nada-ku, Kobe-shi, Hyogo 657-8501, Japan \\ ${ }^{* * *}$ Department of Chemical Science and Engineering, Kobe University \\ 1-1, Rokkodai-cho, Nada-ku, Kobe-shi, Hyogo 657-8501, Japan \\ (Received : February 5, 2016)
}

\begin{abstract}
A flow experiment of a dilute suspension and aggregation/dispersion analysis of fine particles in a non-uniform shear field were performed. We investigated the dispersion characteristics in a microchannel with an abrupt contraction section. The dispersed particles and dispersion media were polystyrene and aqueous glycerine, respectively. The particle diameter and solid volume fraction were $3.5 \mu \mathrm{m}$ and 0.001 , respectively. Citric acid $(0.5$ and $1.0 \mathrm{wt} \%)$ was added as a non-ionic salt in order to investigate the effect of the aggregational force. The suspension was introduced into the microchannel with a syringe pump at a volume rate of $0.3 \mu 1 \cdot \mathrm{min}^{-1}$. The aggregation/dispersion characteristics of the fine particles were observed with a microscope and analyzed by image analysis. We observed the breakup and re-aggregational behavior of the fine particles in a dilute suspension. The cumulative frequencies of fine particles fluctuated through the channel. From the results, we posit the possibility that the non-uniformity of ions affect the aggregational characteristics of fine particles in a shear flow.
\end{abstract}

Key Words: Microchannnel / Abrupt contraction / Suspension / Particle dispersion / Polymer composites

\section{INTRODUCTION}

Polymer composites containing fine particles (e.g., silica) have attracted considerable attention because such composites exhibit a wide range of functionalities with applications ranging from bone tissue engineering to aerospace components. These composites are made from suspensions that disperse particles in a molten polymer. However, the dispersion of fine particles in a polymer is not an easy process to achieve. The fine particles have a high specific surface area, and consequently the interparticle energy resulting from the surface energy becomes relatively large. Thus, the fine particles easily aggregate with each other and form larger clusters. Since the functionality of the polymer composites depends on the particle dispersion, it is essential to control the dispersion characteristics of particles in a molten polymer.

To disperse particles in polymers, a kneading extruder with rotors is often used, and high shear stresses are applied to break up the clusters with the use of the rotors. However,

$\dagger$ E-mail: masuda@kobe-kosen.ac.jp shear stresses vary in time and space (different for different locations) in the extruder. Consequently, the particle dispersion in the polymer composites is non-uniform over both space and time, and this makes it very difficult to determine the dispersion characteristics of the particles in such extruders via experimental studies.

Particle behavior in solid-liquid suspensions is affected by several internal and external factors. Smoluchowski ${ }^{1)}$, Swift and Friedlander ${ }^{2)}$, and Higashitani et $a l^{3-5)}$ have proposed the coagulation model of fine particles in suspensions. On the other hand, Usui ${ }^{6}$ ) has established a thixotropy model that considers the shear breakup of clusters in suspensions and has suggested a method for predicting the rate of change in the cluster size and bulk suspension viscosity. This model has been applied and validated for several polymer composite systems. ${ }^{7,8)}$ The model suggested by Usui can estimate the mean number of particles in a cluster. Since, the existence of large clusters severely affects the function of the polymer composites, Hasegawa et al. ${ }^{9,10)}$ have further developed Usui's model for estimating the cluster size distributions; however, their model can estimate particle dispersion characteristics 
only for a simple shear flow. In other previous studies ${ }^{11,12)}$, a novel thixotropy model was developed for estimating the cluster size under conditions of an unsteady non-uniform flow. With this model, the time-varying cluster size distributions can be locally obtained by considering the local balance of Brownian and shear coagulations and the shear breakup of clusters. Masuda et al. ${ }^{11)}$ have confirmed that this model is sufficiently accurate for steady non-uniform flows by comparing the modeling results with the experimental data obtained for a flow with a backward-facing step.

We previously numerically analyzed a dilute suspension in a flow between two coaxial cylinders. ${ }^{13)}$ In that study, we calculated the aggregation/dispersion characteristics of fine particles in a shear flow when the shear rate was changed drastically. The flow development time of the cluster size profile was longer than that of the velocity profile. In addition, the local viscosity depended on the local cluster size characteristics and the development behavior of the velocity profile depended on the local viscosity. We also previously used a microchannel ${ }^{11)}$ in an experimental and numerical approach to identify the aggregation/dispersion characteristics in a non-uniform shear flow; however, in that analysis, the experiment was performed at only the region of shear rate change, and the re-aggregation behavior was not examined.

In this study, we elucidate the relationship between the velocity field and the cluster size distribution in a nonuniform shear flow. Additionally the effect of adding salt on the cohesive force and the aggregational characteristics were analyzed.

\section{EXPERIMENTAL METHODS}

In this study, a micro-channel, which made in PDMS, was used for micro fluid experiment. The width and depth of the micro-channel was 400 and $100 \mu \mathrm{m}$. A rib as width was $200 \mu \mathrm{m}$ was set at the side of the channel. Fig. 1 shows the observation area of the microchannel used in this experiment. The area was the inlet of the rib where an abrupt contraction flow occurred. The observation area was from $y / H=-1.0$ to 1.4 in a streamwise direction.

Polystyrene particles $(3.5 \mu \mathrm{m})$ were used as the dispersion particles. Aqueous glycerol was used as the dispersion media. The density of the media was equal to that of the dispersion particle. The solid volume fraction was 0.01 . Citric acid 0.5 and $1.0 \mathrm{wt} \%$ was added as a non-ionic salt. For preparing the suspension, polystyrene particles and a small amount of glycerine were stirred and excessively large clusters were dispersed. Glycerine and water were then added in order to equalize the density of the dispersion media and dispersed particles.

Fig. 2 shows the experimental apparatus. The microchannel was connected to the syringe pump by tubing. The prepared suspension, at a volume rate of $0.3 \mu \mathrm{l} \cdot \mathrm{min}^{-1}$, was introduced into the micro-channel by using a syringe pump. The observation area in the depth direction was the mid- point of the microchannel and was detected as the mid-point between the focusing position on the upper and lower plates of the microchannel. The depth of field was $4.2 \mu \mathrm{m}$. The numerical aperture (NA), wavelength of the LED light source, and the refractive index of glycerine were $0.28,450 \mathrm{~nm}$, and 1.45 , respectively. The behavior of the suspension was observed by microscope via transmitted light, using M PLAN APO 20x (Mitutoyo) and MT-40 (Mitutoyo) as the objective and imaging lenses, respectively. The observed image was captured by a CMOS camera Flea3 (Point Gray), using a frame rate and exposure time of $60 \mathrm{fps}$ and $10 \mathrm{~ms}$, respectively.

The velocity field was analyzed by the Particle Image Velocimetry (PIV) method. The aggregational/dispersion characteristics were analyzed by image analysis using OpenCV. In the image analysis for the aggregation characteristics, focused clusters were extracted and the number of particles in the clusters was calculated from the pixel area using Simha's cell model. ${ }^{14)}$

In Simha's cell model, clusters were stored in imaginary cells, and the cluster size $d_{k}[\mathrm{~m}]$ was calculated as follows:

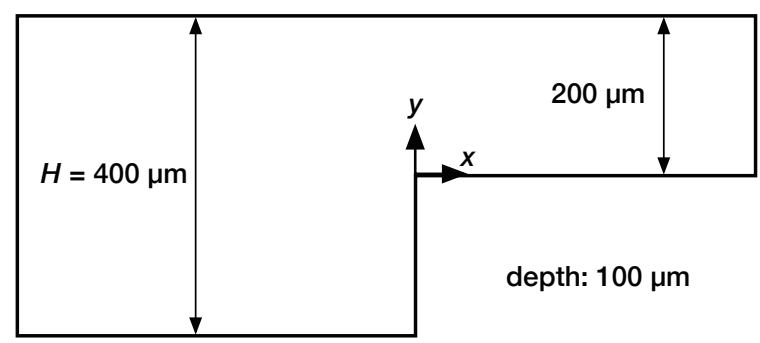

Fig. 1. Observation area.

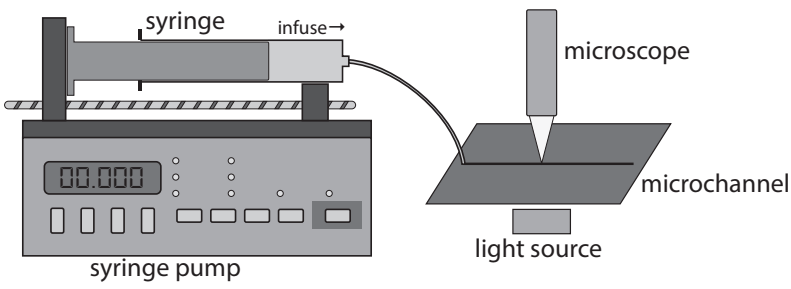

Fig. 2. Experimental apparatus 


$$
d_{k}=d_{0}\left(\frac{k}{1-\varepsilon}\right)^{\frac{1}{3}}
$$

To estimate the void fraction in a cluster, the follow equation was applied, as proposed by Usui:

$$
\varepsilon=\varepsilon_{\max }\left(1-k^{-0.4}\right)
$$

Here, $\varepsilon_{\max }$ denotes the maximum void fraction:

$$
\varepsilon_{\max }=1-\frac{\phi}{\phi_{\mathrm{p}, \max }} .
$$

Here, $\phi_{\mathrm{p}, \max }$ is the maximum packing fraction $(=0.68)$. To calculate the local aggregation/dispersion characteristics of fine particles, microscopic images captured by optical microscope were converted to binary images. Morphological operations were used on the binary images to remove unfocused clusters that were out of the depth of field. The area was calculated from each detected cluster. The equivalent diameter of each cluster was then estimated from the calculated area. The number of particles in a cluster was estimated from the estimated equivalent diameter of the detected clusters by using Simha's cell model. The mean number of particles in a cluster was calculated as follows:

$$
N_{\mathrm{ave}}=\frac{\sum_{k=1}^{k_{\max }} n_{k} \cdot k}{\sum_{k=1}^{k_{\max }} n_{k}} .
$$

The maximum number of particles in a cluster, $k_{\max }$, was 30 . In this way, clusters in the observational area were extracted, and the local aggregational characteristics and space distributions were calculated from the extracted clusters. The observational area for extracting clusters was $72 \times 19 \mu \mathrm{m}$. Six hundred or more clusters were used to estimate cluster size. The error was not more than $5 \%$.

\section{RESULTS AND DISCUSSION}

The velocity vector and cross-sectional velocity profile of the suspension with no citric acid are shown in Figs. 3 and 4, respectively. There was no difference in the velocity profile between the concentrations of citric acid in the suspension, indicating that the size of the clusters did not affect the followup behavior of clusters in the flow. In the observed area, shear rate increased in the abrupt contraction section. The Reynolds number of the flow was about $10^{-3}$. The Reynolds number was defined as follows:

$$
R e=\frac{\rho v_{x} H}{\mu}
$$

where $\rho, v_{x}, H$, and $\mu$ are the density of suspension, streamwise velocity, characteristic length, and viscosity of the suspension, respectively. The characteristic length was the width of the microchannel in this study. From Fig. 4, the shapes of the velocity profiles were almost equal at $x / H=0.23$, 0.60 , indicating that the velocity field was fully developed immediately after the abrupt contraction. This means that the shear rate of the suspension was constant after $x / H=0.23$ in the same $y / H$ position. The shear rates were $2.4 \mathrm{~s}^{-1}$ at a region near the wall $(y / H=0.075)$ and $0.5 \mathrm{~s}^{-1}$ at the center of the channel $(y / H=0.275)$.

Fig. 5 shows cross-sectional profiles of the mean number of particles in a cluster. In Fig. 5a, the mean number of particles in a cluster, i.e., the mean cluster size, took a maximum value estimated to be 4 at $x / H=-0.10$ before the contraction. After the contraction, the mean number of particles in a cluster decreased to almost 3.8 , indicating that clusters broke up

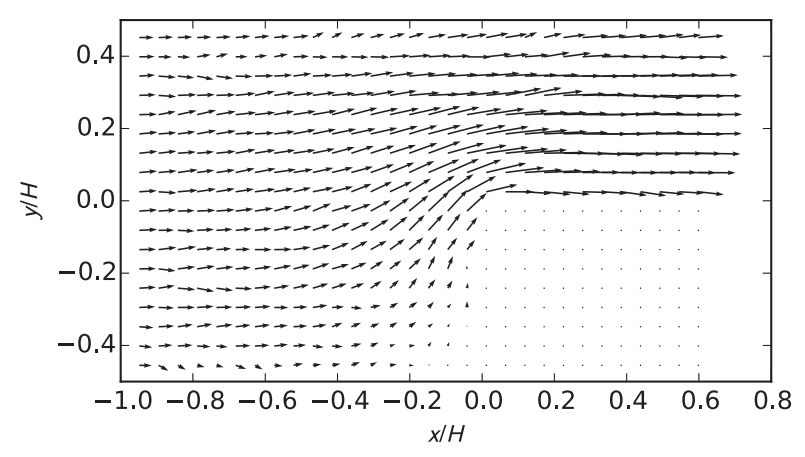

Fig. 3. Velocity vector.

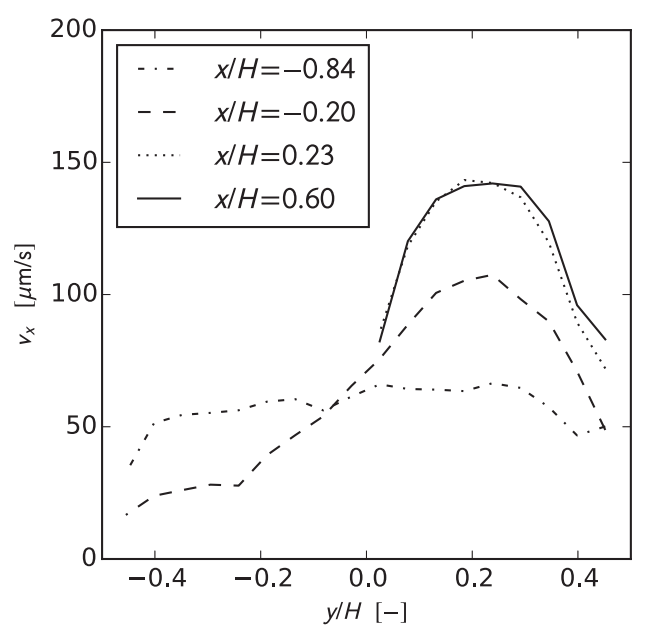

Fig. 4. Velocity profile. 
by application of shear at the abrupt contraction section. In Fig. 5b, the maximum value of the mean number of particles in a cluster is greater than that of Fig. 5a, indicating that the concentration of citric acid affects the re-aggregation process in a shear field.

Fig. 6 shows the streamwise profile of the mean number of particles in a cluster at $y / H=0.075$ and 0.275 . In the case of the lower concentration of citric acid $(0.5 \mathrm{wt} \%)$, the mean cluster size decreased after the channel contraction at a region near the wall, as shown in Fig. 6a. On the other hand, with the higher citric acid concentration (1.0 wt \%), the mean cluster size did not change in the flow at the region near the wall $(y / H=0.075)$. This indicates that the cohesive force of particles due to the addition of citric acid affects the aggregation/dispersion behavior of fine particles. At a region in the center of the channel, the mean cluster size did not change dramatically with the lower concentration of citric acid (0.5 wt\%), as shown in Fig. 6b. On the other hand, with the higher citric acid concentration (1.0 wt $\%)$, the mean cluster size was not constant, and there was a tendency to increase in size at the low shear rate region of the center of the channel for $y / H=0.275$. These results showed that the soft-aggregated cluster was able to break up and re-aggregate in a non-uniform shear field.

Fig. 7 shows the cumulative frequency of the clusters at the near wall region for $y / H=0.075$. In this region, a drastic change in the cumulative frequency of the flow could not be confirmed for either citric acid concentration, although the concentration of small clusters composed of about one to three particles slightly increased at a citric acid concentration of $0.5 \mathrm{wt} \%$, as shown in Fig. 7a. This indicates that some clusters broke into small clusters at the high shear rate region near the

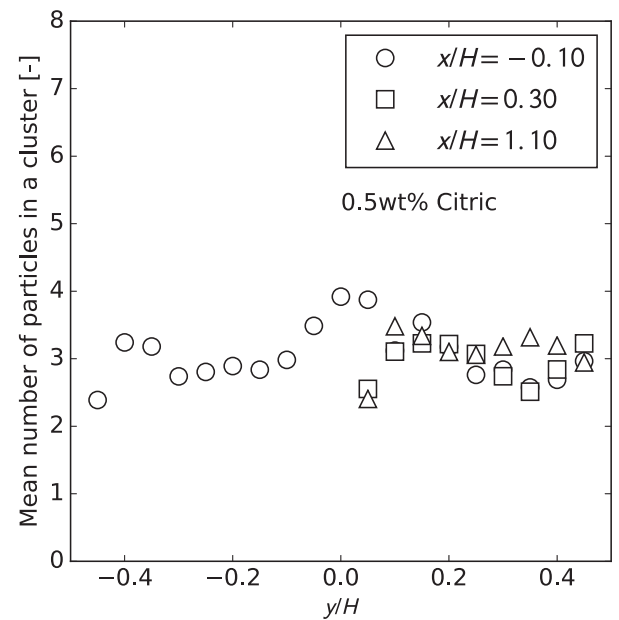

(a)

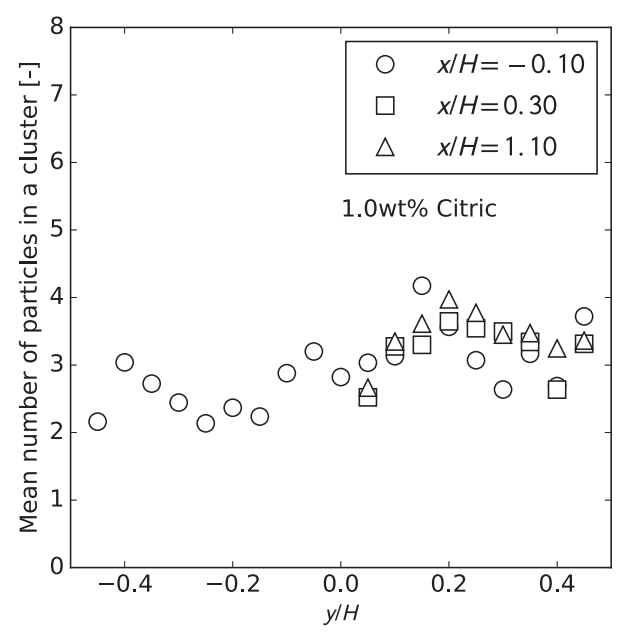

(b)

Fig. 5. Cross-sectional profile of mean number of particles in a cluster with (a) $0.5 \mathrm{wt} \%$ and (b) $1.0 \mathrm{wt} \%$ citric acid.

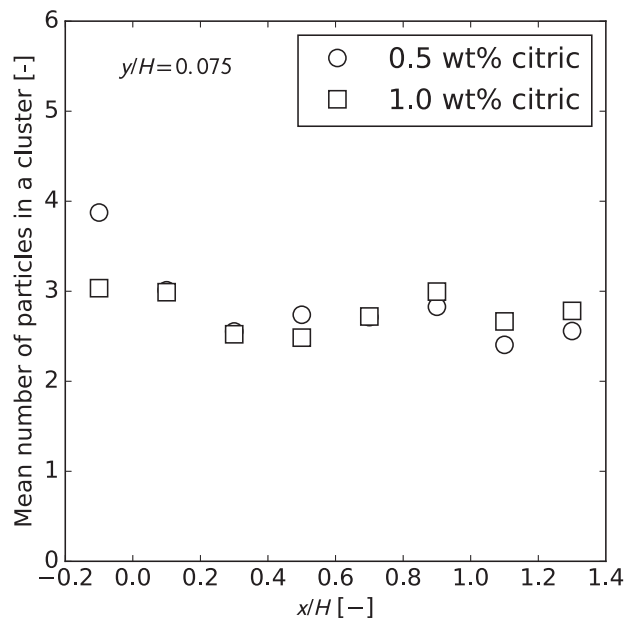

(a)

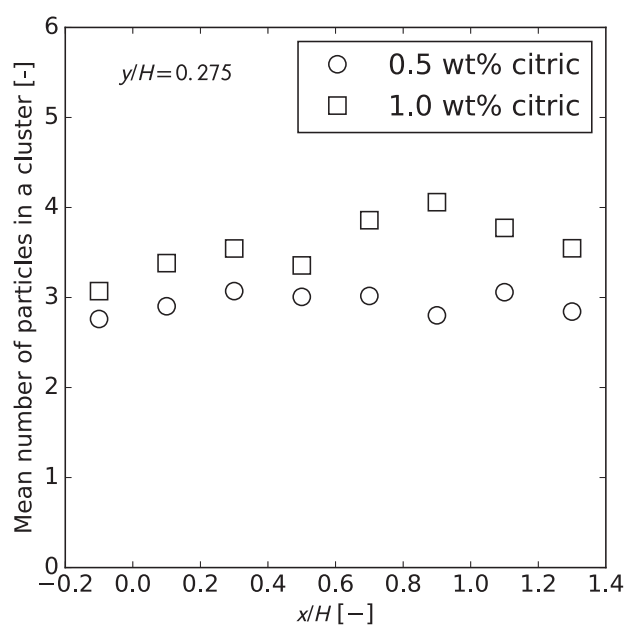

(b)

Fig. 6. Streamwise profile of the mean number of particles in a cluster for $y / H=$ (a) 0.075 and (b) 0.275 . 
wall.

Fig. 8 shows the cumulative frequency of clusters in the center of the channel for $y / H=0.275$. In this region, the cumulative frequencies were not constant in the flow, the shear rate was less than the region near the wall, and the cohesive force due to citric acid was greater than the shear stress. As a result, the local cluster size distribution fluctuated at the lower shear region. The overall aggregation/dispersion behavior showed an increasing tendency, as shown in Fig. 6, although the local cluster size distribution did not show a simple increase. These results suggest that this behavior was affected by a non-uniform ion distribution. Colliding or broken clusters disturbed the suspension, causing the non-uniformity in ion distribution. It is probable that the non-uniformity in the ion distribution intensified the nonlinearity of the increasing mean cluster size.

\section{CONCLUSION}

We performed a flow experiment in a microchannel and analyzed aggregation/dispersion by using image analysis. The mean number of particles in a cluster changed in the fully developed shear flow. This showed the difference between flow development time of a cluster size profile and that of the velocity profile and confirms the validity of previous numerical results. ${ }^{13)}$

From the aggregation/dispersion characteristics obtained by using image analysis, we observed that breakup and reaggregation behaviors occur in a non-uniform shear flow. The addition of citric acid as a salt caused streamwise variations of the cluster size distribution and cumulative frequency, indicating that the valance between the cohesive force and shear stress caused fluctuations in the aggregation/dispersion

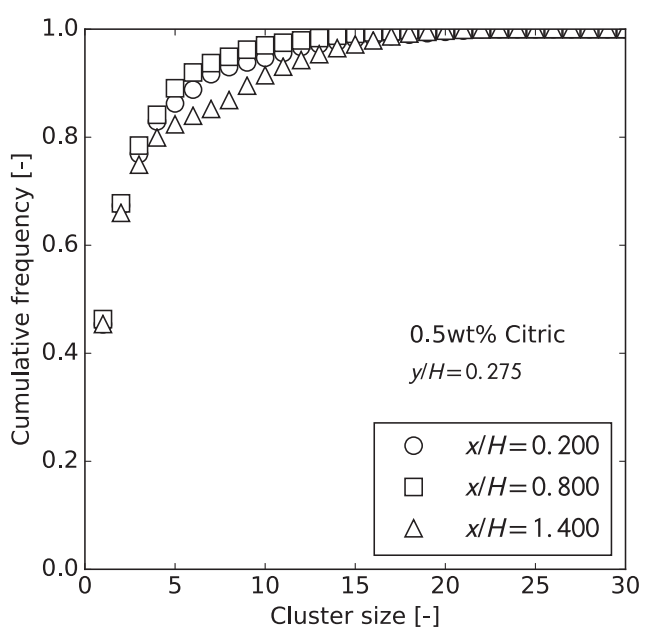

(a)

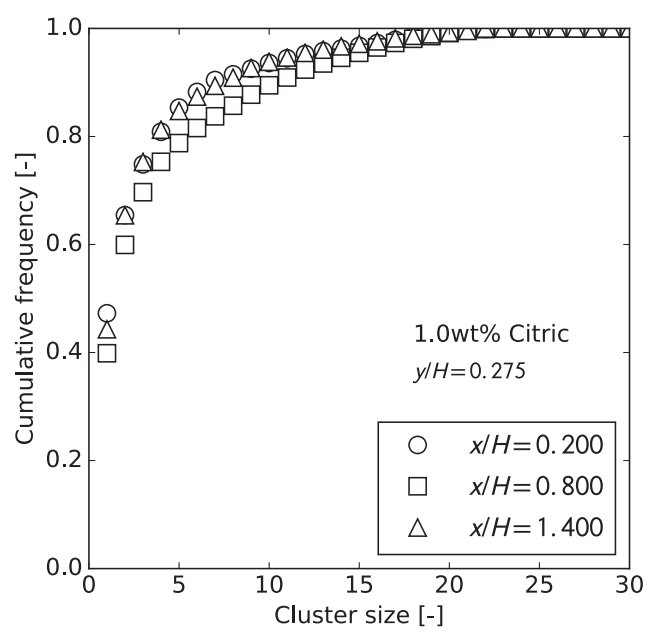

(b)

Fig. 8. Cumulative frequency at $y / H=0.275$ with (a) $0.5 \mathrm{wt} \%$ and (b) $1.0 \mathrm{wt} \%$ citric acid.
Fig. 7. Cumulative frequency at $y / H=0.075$ with (a) $0.5 \mathrm{wt} \%$ and (b) $1.0 \mathrm{wt} \%$ citric acid. 
characteristics. We believe that our findings can contribute to the process such as coating using dilute suspensions.

\section{ACKNOWLEDGMENT}

This work was supported by JSPS KAKENHI Grant Number 26820052.

\section{NOMENCLATURE}

$d_{0} \quad$ Diameter of primary particle

$\mathrm{m}$

$d_{k} \quad$ Diameter of cluster composed of $k$ particles m

$H$ Width of microchannel m

$k \quad$ Number of particles in a cluster

$k_{\max } \quad$ Maximum number of particles in a cluster

$N_{\max } \quad$ Mean number of particles in a cluster

$n_{k} \quad$ Concentration of a cluster composed of $k$ particles

Re Reynolds number

$v \quad$ Velocity

$x, y \quad$ Coordinate

$\mathrm{m} \cdot \mathrm{s}^{-1}$

$\varepsilon \quad$ Void fraction

$\varepsilon_{\max } \quad$ Maximum void fraction

$\mu \quad$ Viscosity of suspension

$\rho \quad$ Density of suspension

$\phi \quad$ Solid Volume fraction

$\phi_{\mathrm{p}, \max }$ Maximum packing fraction

\section{REFERENCES}

1) Smoluchowski Mv, Z Phys Chem, 92, 129-168, (1917).

2) Swift DL, Friedlander SK, J Colloid Sci, 19, 7, 621-647, (1964).

3) Higashitani K, Tanaka T, Matsuno Y, J Colloid Interface Sci, 63, 3, 551-560, (1978).

4) Higashitani $\mathrm{K}$, Ogawa R, Hosokawa G, Matsuno Y, $J$ Chem Eng Jpn, 15, 4, 299-304, (1982).

5) Higashitani K, Iimura K, Sanda H, Chem Eng Sci, 56, $2927-$ 2938, (2001).

6) Usui H, Kagaku Kogaku Ronbunshu, 25, 3, 459-465, (1999).

7) Usui H, Li L, Kinoshita S, Suzuki H, J Chem Eng Jpn, 34, 3, 360-368, (2001).

8) Mustafa, Usui H, Shige I, Suzuki H, Kobayashi T, Miyazaki Y, Ioroi T, Yasuda K, J Chem Eng Jpn, 37, 1, 31-39, (2004).

9) Hasegawa E, Suzuki H, Kameyama K, Komoda Y, Usui H, Adv Powder Technol, 20, 2, 139-144, (2009).

10) Hasegawa E, Suzuki H, Komoda Y, Usui H, Nihon Reoroji Gakkaishi (J Soc Rheol, Jpn), 37, 4, 191-198, (2009).

11) Masuda K, Suzuki H, Komoda Y, Hidema R, Nihon Reoroji Gakkaishi (J Soc Rheol, Jpn), 41, 2, 75-81, (2013).

12) Masuda K, Suzuki H, Komoda Y, J Chem Eng Jpn, 46, 8, 524-529, (2013).

13) Masuda K, Suzuki H, Komoda Y, Hidema R, Nihon Reoroji Gakkaishi (J Soc Rheol, Jpn), 43, 3-4, 85-92, (2015).

14) Simha R, J Appl Phys, 23, 9, 1020-1024, (1952). 